\title{
Influence of the Static Sample Temperature on the Mass Spectra of Alkali Halides and Metals Obtained with Pulsed Laser Induced Ion Generation*
}

\author{
B. Schueler** \\ University of Manitoba, Department of Physics, Winnipeg, Manitoba R3T 2N2, Canada \\ P. K. D. Feigl** and F. R. Krueger \\ Max-Planck-Institut für Kernphysik, Abteilung Kosmophysik, Heidelberg \\ Z. Naturforsch. 38a, 1078-1083 (1983); received July 4, 1983
}

Experimental investigations of the interdependence between the ion yield and the static sample temperature at a fixed laser irradiance, between the ion yield and the laser irradiance at different static sample temperatures and between the ion detection threshold irradiance and the static sample temperature are reported. Laser wavelengths of $532 \mathrm{~nm}, 355 \mathrm{~nm}$ and $266 \mathrm{~nm}$ have been used to obtain the data. The laser pulse duration was $10 \mathrm{~ns}$. Experimental results for alkali halides and metals are reported and compared. A strong dependence of the experimental parameters mentioned above on the static sample temperature has been observed for alkali halides. In the case of metals only minor effects have been found. In any case the different laser irradiation wavelengths do not significantly effect the experimental results.

\section{Introduction}

Various studies on ion formation by pulsed laser irradiaton of metals and inorganic and organic substances have been published $[1,2,3]$. A variety of ion formation models has been suggested $[4,5,6]$. These models do not explicitly contain parameters such as laser pulse duration or laser wavelength, the temperature, however, is one common feature of all the models. With this general situation in mind we started experimental investigations on the effect of the static sample temperature on the mass spectra of metals and alkali halides. We expected this to provide a good test of theoretical models of the ion formation process.

Three types of measurements have been performed: the dependence of the ion yield i) on the static sample temperature at fixed laser irradiances, ii) on the laser irradiance at fixed static sample temperatures, and iii) the dependence of the threshold irradiance for ion detection on the static sample temperature.

* Supported by the Deutsche Forschungsgemeinschaft (DFG) and the Federal Department of Research and Technology through VDI, Berlin.

** Formerly: Institut für Biophysik, Goethe-Universität, Theodor-Stern-Kai 7, D-6000 Frankfurt (Main) 70, West Germany.

Reprint requests to Dr. F. R. Krueger, Max-Planck-Institut für Kernphysik, Abteilung Kosmophysik, Saupfercheckweg, D-6900 Heidelberg.

\section{Experimental}

The experimental set-up used in the investigations is the LAMMA $^{\circledR} 1000$ instrument, as described in [7]. The laser used is a Q-switched TEMoo-mode Nd-YAG laser with a pulse duration of about $10 \mathrm{~ns}$ and $1064 \mathrm{~nm}$ wavelength. By means of frequency multiplication it is also possible to operate at the wavelengths of $532 \mathrm{~nm}, 355 \mathrm{~nm}$ and $266 \mathrm{~nm}$. The laser irradiances used to obtain the mass spectra were between $10^{8}$ and $10^{9} \mathrm{~W} / \mathrm{cm}^{2}$.

High resolution sample observation with light and dark field illumination is possible by positioning a 0.6 numerical aperture (n.a.) microscopic objective in front of the sample (Figure 1a). In this position the sample spot to be investigated can be selected. Figure $1 \mathrm{~b}$ shows the LAMMA 1000 sample stage in the position for laser irradiation (sample illumination), and low resolution sample observation at an angle of $30^{\circ}$ to the spectrometer axis. A few micron spot on the sample is irradiated by the laser beam through a 0.2 n. a. ultraviolet corrected monochromate objective. The ions produced are extracted perpendicularly to the sample surface by a $3 \mathrm{kV}$ acceleration voltage of the first element of the ion lens, and the ions are mass separated by a linear time-of-flight detection system. The positioning accuracy between the two positions of the sample stage is better than $1 \mu \mathrm{m}$.

In order to record mass spectra of samples at different static sample temperatures $\left(T_{\mathrm{s}}\right)$ a special 


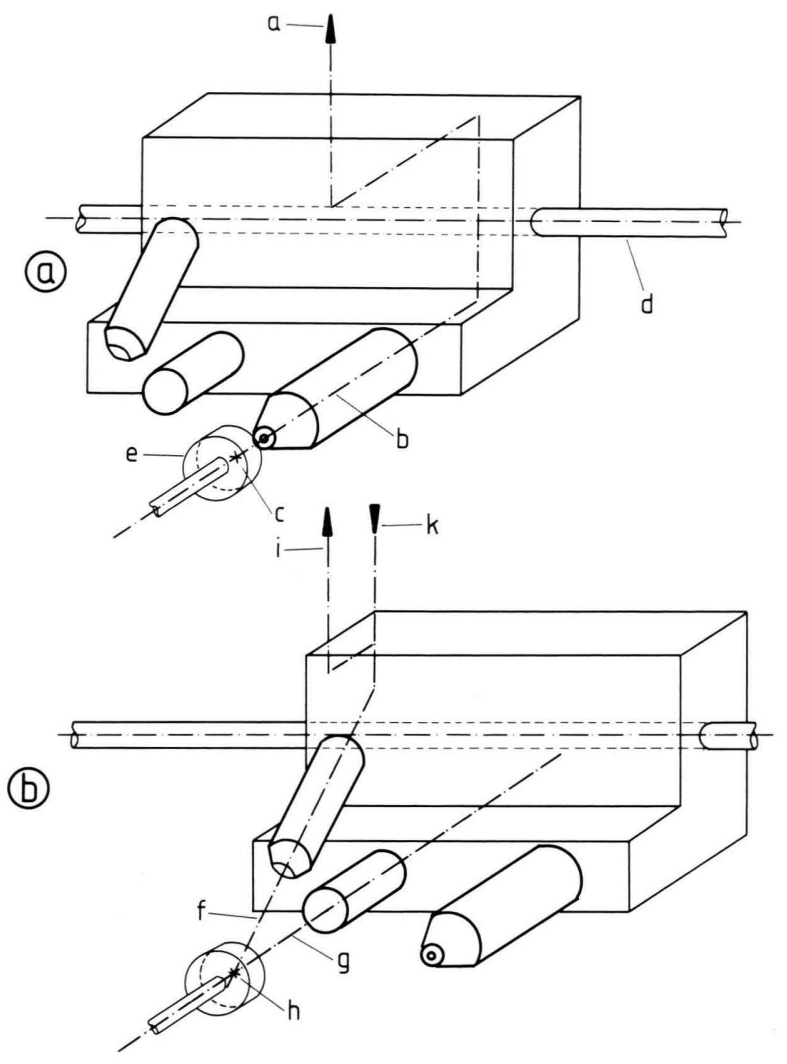

Fig. 1. The LAMMA ${ }^{(B)} 1000$ sample chamber in (a) the observation position and (b) the analysis position. a) high resolution observation, b) high resolution microscope objective, c) selected sample spot, d) axis of translational movement, e) sample, f) optical axis of the uv-monochromate, g) spectrometer axis, h) irradiated sample spot $(=c), \mathrm{i})$ low resolution observation, $\mathrm{k})$ incident laser beam.

\section{Results}

\subsection{Dependence of the ion yield on the static sample temperature at fixed laser irradiance}

In a first set of experiments the mass spectra of positive ions of alkali halides and metals have been recorded for fixed laser irradiances at various static sample temperatures $\left(T_{\mathrm{s}}\right)$. In order to provide low statistical deviations of the ion yield obtained in different mass spectra and to optimize cluster ion detection, the laser irradiance was always chosen to be twice the threshold irradiance for ion detection at room temperature, i.e. about $10^{9} \mathrm{~W} / \mathrm{cm}^{2}$. The ion yields $\left(y^{+}\right)$have been determined by averaging the yields of at least ten mass spectra.

The salts investigated have been cesium halides and lithium fluoride, electrosprayed on Ni-wires. A strong dependence of $y^{+}$on $T_{\mathrm{s}}$ has been observed for all salts measured. An increase of $T_{\mathrm{s}}$ gives rise to an increase of $y^{+}$as shown in Figure 2. Initially the ion yield of the salt cation increases only slightly up to $T_{\mathrm{s}} \approx 500 \mathrm{~K}$. At higher $T_{\mathrm{s}}$ a very steep increase in $y^{+}$is observed. The ratios of the ion yields at the maximum $T_{\mathrm{s}}(\approx 800 \mathrm{~K})$ to those at room temperature are about ten.

The plots in Fig. 2 are based on experiments performed at $355 \mathrm{~nm}$ laser wavelength. They also represent the general temperature dependence of the cation yield of salts taken at $532 \mathrm{~nm}$ and $266 \mathrm{~nm}$.

In the mass spectra of alkali halides obtained under the above conditions, cluster ions (e.g. $\mathrm{Cs}_{2} \mathrm{I}^{+}$)

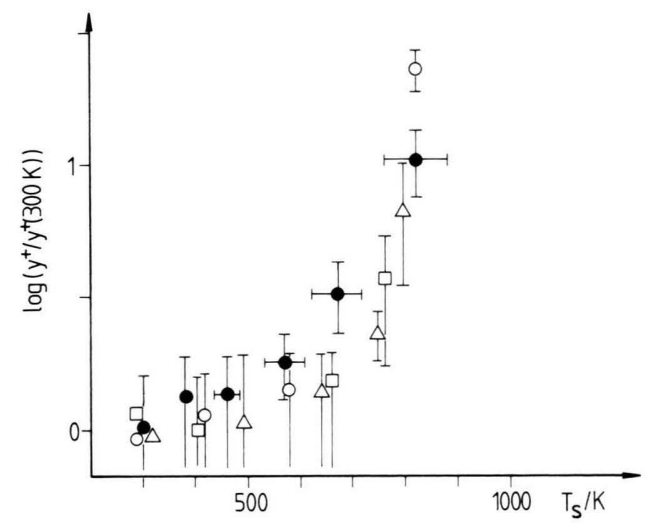

Fig. 2. Dependence of the salt cation yield from $\operatorname{LiF}(0)$, $\mathrm{CsF}(\triangle), \mathrm{CsCl}(\square)$, and $\mathrm{CsI}(\bullet)$ on the static sample temperature $T_{\mathrm{s}}$ at fixed laser irradiances. The error bars shown for CsI are representative of the other samples. The ion yield for any substance is normalized to its value at room temperature. 
have also been detected. The dependence of the cluster ion yield $y_{2}^{+}$on $T_{\mathrm{s}}$ is rather different from that observed for $y^{+}$. For $T_{\mathrm{s}}$ up to $500 \mathrm{~K}, y^{+}$does not change significantly. For higher $T_{\mathrm{s}}$ it decreases to values below the detection sensitivity of the mass spectrometer. The $T_{\mathrm{s}}$ at which $y_{2}^{+}$vanishes is also the temperature at which a noticeable increase in $y^{+}$ is observed.

If the sample temperature after heating is lowered to room temperature, cluster ion formation does not recommence. To verify this, CsI samples have also been treated through a temperature cycle outside the sample chamber under ambient conditions. The results resembled those described above, i.e. annealed samples exhibited a drastically diminished yield of cluster ions.

In the case of metals a very similar qualitative dependence of the metal monomer ion yield $y^{+}$on $T_{\mathrm{s}}$ is observed. As shown in Fig. 3 for Pt, Cu, Fe, and $\mathrm{Ni}, y^{+}$also increases with increasing $T_{\mathrm{s}}$. However, when approaching the melting point of the metal, the increase in $y^{+}$is not as steep as the corresponding yield increase observed with alkali halide samples. It is observed that there is no interdependence between the $T_{\mathrm{s}}$-behaviour of the metal ion yield and the laser wavelength.

An important difference is found for the temperature dependence of the ion yield for metal cluster ions compared to that of alkali halide clusters (Figure 4). The yield of metal cluster ions does not disappear with increasing $T_{\mathrm{s}}$ but increases with increasing $y^{+}$of the metal monomer ion.

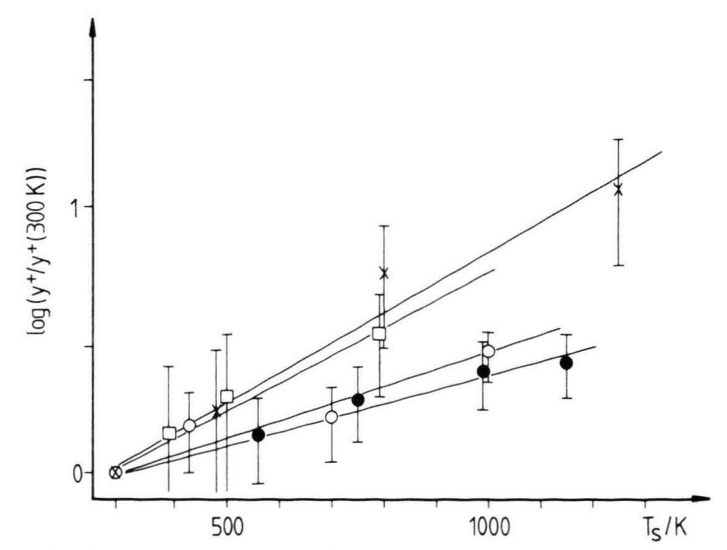

Fig. 3. Dependence of the normalized metal monomer ion yield on $T_{\mathrm{s}}$ at fixed laser irradiances for $\mathrm{Pt}(\times), \mathrm{Cu}(\square)$, $\mathrm{Fe}(0), \mathrm{Ni}(\bullet), \lambda=532 \mathrm{~nm}$.

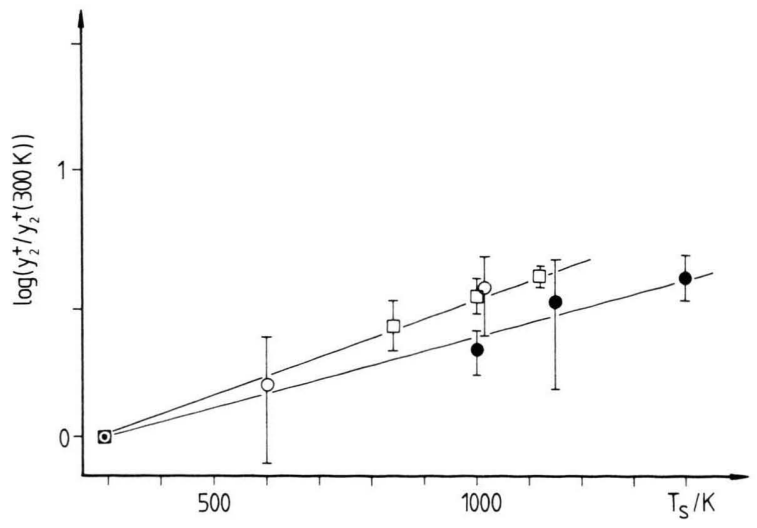

Fig. 4. Dependence of the normalized metal cluster ion yield on $T_{\mathrm{s}}$ at fixed laser irradiances for $\mathrm{Cu}(\square), \mathrm{Fe}(0)$, $\mathrm{Ni}(\bullet), \lambda=532 \mathrm{~nm}$.

\subsection{Dependence of the ion yield on the laser irradiance at fixed static sample temperatures}

The dependence of the ion yield on the laser irradiance $\left(A^{+}\right)$for various fixed static sample temperatures was also investigated. The ion yields reported are averages over at least ten mass spectra. $A^{+}$was varied by means of filters placed into the laser optical beam path. Every laser shot was energy monitored.

The variation in $y^{+}$with $A^{+}$is shown in Figs. $5 \mathrm{a}-\mathrm{c}$ for CsI at three $T_{\mathrm{s}}$ values. The dependence of $y^{+}$on $A^{+}$can be empirically approximated by $\log \left\{y^{+} / y_{0}\right\} \sim$ $\left\{A^{+}\right\}^{-1}$ for irradiances above the threshold irradiance $A_{\mathrm{th}}^{+}$for ion detection. The decrease in the slope of the fitting lines for increasing $T_{\mathrm{s}}$ only results from the normalization of the irradiances to $A_{\text {th }}^{+}$for ion detection at $T_{\mathrm{s}}=600 \mathrm{~K}$, because $A_{\text {th }}^{+}$ depends on $T_{\mathrm{s}}$ as described in more detail in section 3.3. When comparing the dependence of $\log \left\{y^{+} / y_{0}\right\}$ on $\left\{A_{\mathrm{th}}^{+}\left(T_{\mathrm{s}}\right) / A^{+}\right\}$, the slopes are about the same (Figures $5 \mathrm{a}-\mathrm{c}$ ). It is also derived from Figs. $5 \mathrm{~b}$ and $5 \mathrm{c}$ that the dependence of $y^{+}$on $A^{+}$ can be described by the same approximation. At $T_{\mathrm{s}}=600 \mathrm{~K}$ no cluster ions could be detected. Thus, the yield ratio $y^{+} / y_{2}^{+}$for salt cations and cluster ions is not affected by $A^{+}$but strongly influenced by changes in $T_{\mathrm{s}}$. The laser wavelength used to obtain the data was $266 \mathrm{~nm}$, but this general behaviour was also observed at $355 \mathrm{~nm}$ and $532 \mathrm{~nm}$.

The dependence of the $\mathrm{Fe}^{+}$-ion yield on $A^{+}$is shown in Fig. 6 for three static sample temperatures. Again the results are very similar to those obtained with salts. 

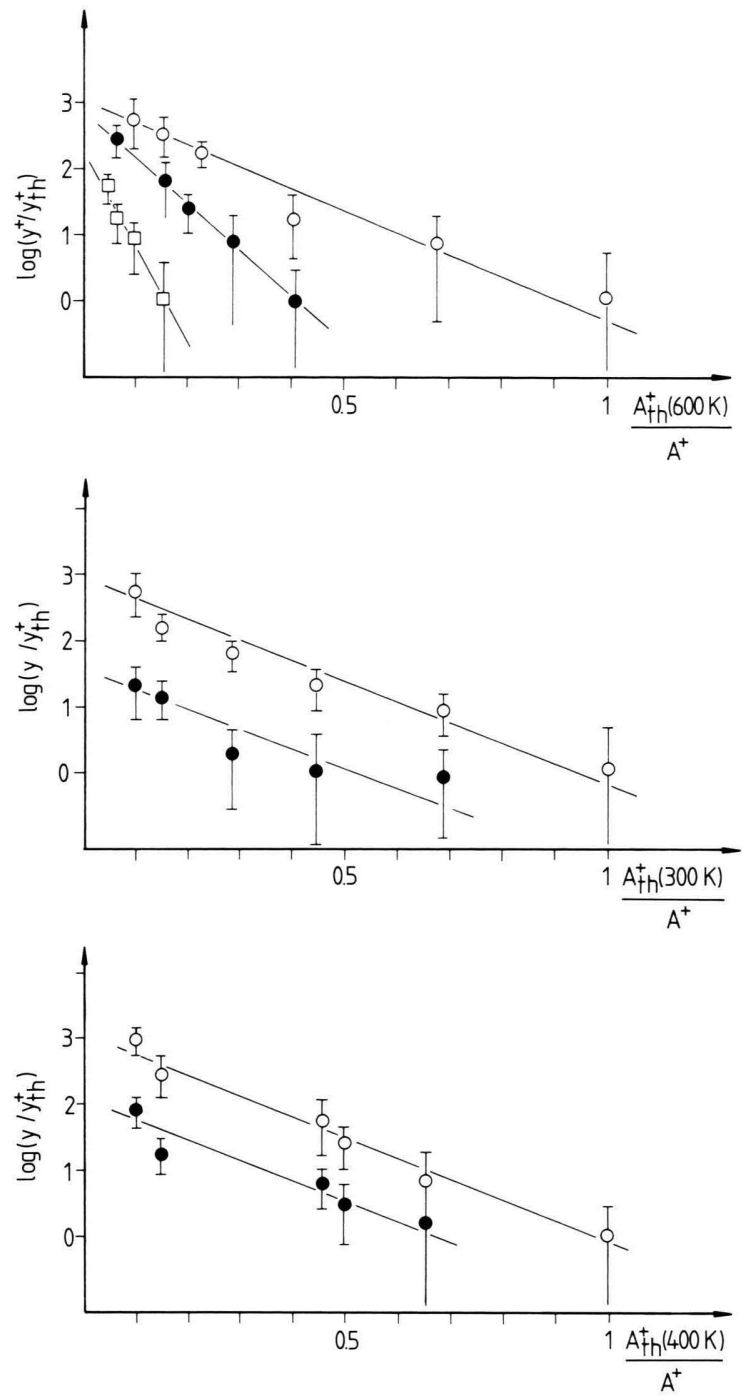

Fig. 5. a) Dependence of $\log \left\{y\left(\mathrm{Cs}^{+}\right)\right\}$for CsI at the laser wavelength of $266 \mathrm{~nm}$ on the inverse laser irradiance obtained at $T_{\mathrm{s}}=300 \mathrm{~K}(\square), 400 \mathrm{~K}(\bullet)$, and $600 \mathrm{~K}(0)$. The laser irradiance are normalized to the threshold irradiance for ion detection at $T_{\mathrm{s}}=600 \mathrm{~K}$. b) Dependence of the $\mathrm{Cs}^{+}(0)$ and $\mathrm{Cs}_{2} \mathrm{I}^{+}(\bullet)$ ion yields on $\left\{A_{\mathrm{th}}^{+}\left(T_{\mathrm{s}}=300 \mathrm{~K}\right)\right\} / A^{+}$ obtained at $T_{\mathrm{s}}=300 \mathrm{~K}$ c) Dependence of the $\mathrm{Cs}^{+}(0)$ and $\mathrm{Cs}_{2} \mathrm{I}^{+}(\bullet)$ ion yields on $\left\{A_{\mathrm{th}}^{+}\left(T_{\mathrm{s}}=400 \mathrm{~K}\right)\right\} / A^{+}$obtained at $T_{\mathrm{s}}=400 \mathrm{~K}$

\subsection{Threshold irradiance for ion detection as a function of the static sample temperature}

In this set of experiments the threshold laser irradiances $\left(A_{\mathrm{th}}^{+}\right)$for ion detection were determined at various static sample temperatures. $A_{\text {th }}^{+}$of the substance in question is defined to be the laser irradiance leading to a $50 \%$ probability of ion detec- tion in the mass spectra. The experiments were performed at the laser wavelengths of $532 \mathrm{~nm}, 355 \mathrm{~nm}$, and $266 \mathrm{~nm}$. Every laser shot was energy monitored.

The dependence of the normalized $A_{\mathrm{th}}^{+}$of cesium iodide on $T_{\mathrm{s}}$ and for the three laser wavelengths mentioned above is shown in Figure 7. The ion detection threshold decreases with increasing $T_{\mathrm{s}}$. The laser wavelength used in the experiments does not significantly influence the $A_{\mathrm{th}}^{+}-T_{\mathrm{s}}$ interdependence. $A_{\text {th }}^{+}$is generally lowered by about a factor of ten at the maximum $T_{\mathrm{s}}$ in comparison to its value at room temperature.

The situation is comparable for metals where a decrease of $A_{\text {th }}^{+}$for ion detection is also observed. In the case of iron, at $T_{\mathrm{s}}=1000 \mathrm{~K}$ the threshold irradiance is about half the value at room temperature. No wavelength dependence can be observed as mentioned for alkali halides.

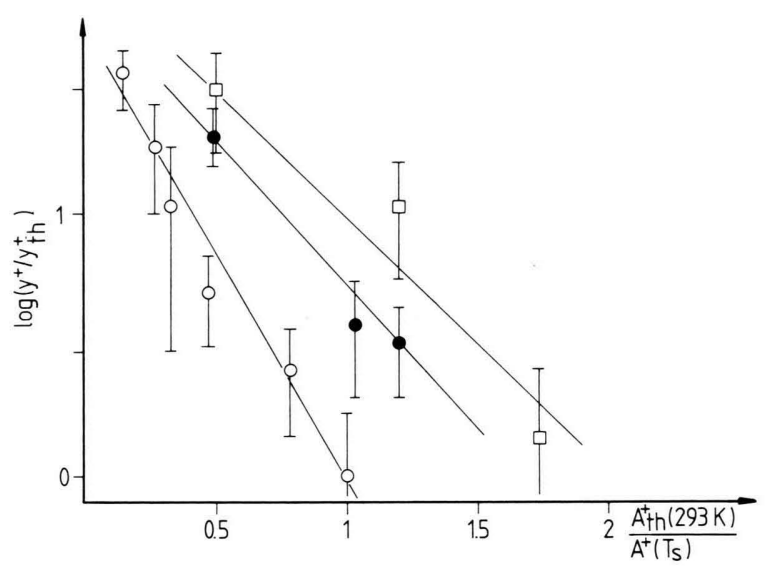

Fig. 6. Dependence of the $\mathrm{Fe}^{+}$ion yield on the laser irradiance at $T_{\mathrm{s}}=293 \mathrm{~K}(0), 900 \mathrm{~K}(\bullet)$, and $1100 \mathrm{~K}(\square)$. The laser irradiances are normalized to the ion detection threshold irradiance at room temperature.

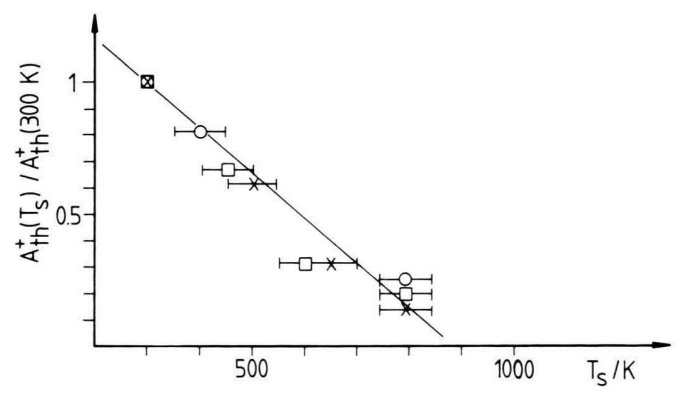

Fig. 7. Dependence of the normalized threshold irradiance $\left\{\boldsymbol{A}_{\mathrm{th}}^{+}\left(T_{\mathrm{s}}\right)\right\} /\left\{\boldsymbol{A}_{\mathrm{th}}^{+}(300 \mathrm{~K})\right\}$ for ion detection of $\mathrm{Cs}^{+}$-ions out of

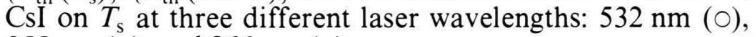
$355 \mathrm{~nm}(\bullet)$, and $266 \mathrm{~nm}(\square)$. 


\section{Discussion}

It has been shown by Jöst et al. (e.g. [4], [10]) that ions created in gasphase reactions (decay of larger molecules, ion-molecule-reactions) are not expected to be significant in the mass spectra obtained under the experimental conditions used. Thus, the "thermal aspects" of fast ion formation induced by pulsed uv-laser irradiation will be discussed in terms of a solid state ion formation process. One interesting question is whether the action of the laser irradiation on the sample is simply a rapid temperature increase $\Delta T_{\text {ind }}$ at the irradiated sample region. If a quasi-reversible isothermal process is responsible for the ion formation from the solid, the ion yield $y^{+}$of one ion type is given by:

$$
y^{+}(T) \sim \exp \{-\Delta H / R T\},
$$

where $\Delta H$ is the enthalpy of formation and $T$ the temperature. Comparing the dependence of $y^{+}$on $T$ with Figs. 5 and 6 , a very similar dependence of $y^{+}$ on $A_{\mathrm{th}}^{+}\left(y^{+} \sim \exp \left\{-B A_{\mathrm{th}}^{+} / A^{+}\right\}\right)$is found, suggesting a laser induced temperature increase $\Delta T_{\text {ind }} \sim A_{\mathrm{th}}^{+} / A^{+}$. From this dependence of $y^{+}$on $A^{+}$, a laser induced temperature increase $\Delta T_{\text {ind }}$ can be estimated as

$$
\frac{y^{+}\left(A_{\mathrm{th}}\right)}{y^{+}\left(n A_{\mathrm{th}}^{+}\right)}=\exp \left\{\frac{-\Delta H}{R}\left[\frac{1}{\Delta T_{\text {ind }}^{0}}-\frac{1}{n \Delta T_{\text {ind }}^{0}}\right]\right\},
$$

where $\Delta T_{\text {ind }}^{0}$ is the laser induced temperature increase at the laser irradiance $A_{\mathrm{th}}^{+}, y^{+}\left(A_{\mathrm{th}}^{+}\right)$the corresponding ion yield and $n \Delta T_{\text {ind }}^{0}$ and $y^{+}\left(n A_{\mathrm{th}}^{+}\right)$the temperature increase and the ion yield at $A^{+}=$ $n A_{\mathrm{th}}^{+}$. For salt cations from CsI (at any static sample temperature) a ratio of $\left\{y^{+}\left(A_{\mathrm{th}}^{+}\right)\right\}:\left\{y^{+}\left(10 A_{\mathrm{th}}^{+}\right)\right\} \approx 10$ is derived from Figure 5. Using $\triangle H\left(\mathrm{Cs}^{+}\right) \approx$ $600 \mathrm{~kJ} / \mathrm{mole}$, this yields a laser induced temperature increase at the ion detection threshold irradiance of $\Delta T_{\text {ind }}^{0}(\mathrm{CsI}) \approx 9500 \mathrm{~K}$.

Applying the same arguments to the dependence of the $\mathrm{Fe}^{+}$-ion yield on $A^{+}$(Fig. 6) and using $\Delta H\left(\mathrm{Fe}^{+}\right) \approx 100 \mathrm{~kJ} / \mathrm{mole}, \Delta T_{\text {ind }}(\mathrm{Fe})$ is found to be about $20000 \mathrm{~K}$.

However, if the laser irradiation of the sample really induces temperature jumps $\Delta T_{\text {ind }}$ as mentioned above, static heating of the sample should simply give rise to an additional contribution $T_{\mathrm{s}}$ to $\Delta T_{\text {ind }}$, thus leading to a "total evaporation temperature" $T_{\text {tot }}=\Delta T_{\text {ind }}+T_{\mathrm{s}}$. Therefore it should be possible to fit the dependence of $y^{+}$on $T_{\mathrm{s}}$ at a fixed $A^{+}$by a straight line dependence of $\ln \left\{y^{+}\right\}$on $T_{\text {tot }}$.

When applying this description to the experimental data for alkali halides (Fig. 2) and using
$\Delta T_{\text {ind }}$ as fitting parameter, it is found that the best approximation to the experimental values is obtained for $\Delta T_{\text {ind }} \approx 4000 \mathrm{~K}$. This temperature, however, is just half the value of $\Delta T_{\text {ind }}^{0}$ determined from the dependence of $y^{+}$on $A^{+}$.

The situation is similar for metals, where a $\Delta T_{\text {ind }}$ of about $6000 \mathrm{~K}$ is suggested for $\mathrm{Fe}$, again lower than the laser induced temperature jump derived above.

The dependence of $A_{\mathrm{th}}^{+}$on $T_{\mathrm{s}}$ may be used as a further test for the thermal ion formation model. Following similar arguments as above, sample irradiation with $A_{\text {th }}^{+}$gives rise to a certain minimum temperature increase $\Delta T_{\mathrm{m}}=\Delta T_{\text {ind }}+T_{\mathrm{s}}$, high enough to enable ion detection. The increase of $T_{\mathrm{s}}$ would therefore decrease the laser induced temperature increase $\left(\sim A_{\mathrm{th}}^{+}\right)$necessary to obtain the fixed value $\Delta T_{\mathrm{m}}$. This expected decrease of $A_{\mathrm{th}}^{+}$with increasing $T_{\mathrm{s}}$ is in qualitative agreement with the experimental results (Figure 7). As shown in Fig. 7 a ratio of $\left\{A_{\mathrm{th}}^{+}\left(T_{\mathrm{s}}=300 \mathrm{~K}\right\} /\left\{A_{\mathrm{th}}^{+}\left(T_{\mathrm{s}}=800 \mathrm{~K}\right\} \approx 10\right.\right.$ is observed. This, however, would be equivalent to $\left\{\Delta T_{\text {ind }}^{0}\left(T_{\mathrm{s}}=300 \mathrm{~K}\right\} /\left\{\Delta T_{\text {ind }}^{0}\left(T_{\mathrm{s}}=800 \mathrm{~K}\right)\right\} \approx 10 . \quad\right.$ As the total "threshold temperature" $\Delta T_{\mathrm{m}}$ should always be the same, this ratio yields:

$$
\frac{\Delta T_{\text {ind }}^{0}\left(T_{\mathrm{s}}=300 \mathrm{~K}\right)}{\left\{\Delta T_{\text {ind }}^{0}\left(T_{\mathrm{s}}=300 \mathrm{~K}\right)\right\}-500 \mathrm{~K}} \approx 10,
$$

and thus $\Delta T_{\text {ind }}^{0}\left(T_{\mathrm{s}}=300 \mathrm{~K}\right) \approx 550 \mathrm{~K}$. For iron, the ratio of the threshold irradiances has been determined to be $\left\{A_{\mathrm{th}}^{+}(300 \mathrm{~K})\right\} /\left\{A_{\mathrm{th}}^{+}(1100 \mathrm{~K})\right\} \approx 2$, thus implying a $\Delta T_{\text {ind }}^{0}\left(T_{\mathrm{s}}=300 \mathrm{~K}\right)$ of about $1600 \mathrm{~K}$. Both temperatures determined in this section are in sharp contrast to the $\Delta T_{\text {ind }}^{0}$ determined before.

The results show clearly that the fast ion formation process induced by pulsed laser irradiation cannot be simply related to a heating process. This can also be stated by the analysis of total evaporation rates, charged-to-neutral ratios and absolute evaporation rates as discussed in $[11,12]$.

The influence of the static sample temperature on the mass spectra of alkali halides, especially the irreversibe breakdown of the cluster ion detection at higher static sample temperatures indicates that the physical and chemical properties of the sample itself are important for the ion formation process. For all materials investigated the action of the laser irradiation on the sample seems to be fundamentally different from and independent of static heating of the sample. 
[1] N. Fürstenau and F. Hillenkamp, Int. J. Mass Spectr. Ion Phys. 37, 135 (1981).

[2] K.-D. Kupka, F. Hillenkamp, and Ch. Schiller, Adv. Mass Spectrom. 8, 935 (1980).

[3] F. R. Krueger and B. Schueler, Adv. Mass Sepctrom. 8, 918 (1980).

[4] B. Jöst, B. Schueler, and F. R. Krueger, Z. Naturforsch. 37 a, 18 (1982).

[5] H. J. Heinen, Int. J. Mass Spectrom. Ion Phys. 38, 309 (1981).

[6] U. Haas, P. Wieser, and R. Wurster, Analyt. Chemie 308, 270 (1981).
[7] P. K. D. Feigl, B. Schueler, and F. Hillenkamp, Int. J. Mass Spectrom. Ion Phys. 47, 15 (1983).

[8] E. Bruninx and G. Rudstam, Nucl. Instrum. Meth. 13, 131 (1961)

[9] B. Schueler, P. K. D. Feigl, F. R. Krueger, and F. Hillenkamp, Org. Mass Spectrom. 16, 502 (1981).

[10] B. Jöst, Diploma Thesis, Frankfurt (Main) 1980.

[11] B. Schueler, P. K. D. Feigl, and F. R. Krueger, Int. J. Mass Spectrom. Ion Phys. 47, 3 (1983).

[12] F. R. Krueger, Z. Naturforsch. 38a, 385 (1983). 